\title{
Relations between the development of patterns of sleeping heart rate and body temperature in infants
}

\author{
S A Petersen, C Pratt, M P Wailoo
}

\begin{abstract}
Overnight patterns of rectal temperature and heart rate were recorded from 119 normal infants at weekly intervals from 7 to about 16 weeks of age. All data were collected in the infants' own homes. As previously reported, different infants developed an adult-like night time rectal temperature pattern abruptly at different ages. When heart rate data were collated by age, there was an apparently gradual fall in sleeping heart rate from 7 to about 14 weeks of age. This was, however, an artefact of data collation. Individual infants showed abrupt falls in heart rate at the time that the adult-like body temperature pattern appeared, but this occurred at different ages in different babies, so when data were collated cross sectionally, an apparently gradual fall resulted. The relation between the developmental changes in sleeping heart rate and rectal temperature was different in boys and girls, with girls showing a more abrupt and greater change in heart rate at the time of development of the adult-like body temperature pattern. Infants whose parents smoked had significantly lower heart rates once the adult-like body temperature pattern had appeared.

(Arch Dis Child Fetal Neonatal Ed 2001;85:F133-F136)
\end{abstract}

Keywords: heart rate; sleeping; body temperature

There are pronounced changes in patterns of sleeping body temperature over the first months of life. We have shown ${ }^{12}$ that babies develop an adult-like night time body temperature pattern (body temperature falling to about $36.2^{\circ} \mathrm{C}$ ) over the course of a few days at somewhere between 2 and 5 months of age. The age of onset of this adult-like pattern is very variable, ranging in normal infants from 7 to about 16 weeks. It occurs even later in some groups, particularly infants with low Apgar scores at birth. ${ }^{3}$ The abruptness of the change is only shown by detailed longitudinal studies of individual infants. If cross sectional data are collated by age, sleeping body temperature appears to fall gradually with age, as an increasing fraction of the babies sampled acquire the adult-like pattern.

Previous studies of heart rate patterns in infants that have passed the newborn stage $\mathrm{e}^{5}$ appear to show a similar progressive fall in sleeping heart rate with age. This gradual decline may also be an artefact of the cross sectional analysis of the data. In this paper therefore we describe detailed longitudinal measurements of sleeping body temperature and heart rate of infants, in order to establish how the two are related. We report that heart rate changes in a similarly abrupt way to body temperature and at the same time, but in a different way, in the two sexes. Furthermore sleeping heart rate in infants is affected by home circumstances, such as parental smoking.

\section{Methods}

A total of 119 babies were studied over three years during 1990-1993. Subjects were recruited soon after birth from babies born in the Leicestershire maternity hospitals. A trained health visitor approached parents, and explained the purpose of the study to them. Over this and a range of other studies, about $40 \%$ of parents approached agreed to the monitoring of their infants. There were no significant differences in demographic variables between those families who agreed and those who did not. Parents gave informed consent to the monitoring of their infants at home one night a week from 6 to at least 16 weeks of age. Ethical permission was obtained for the studies.

Full perinatal data were collected on each infant. A trained health visitor undertook each of the home monitorings. The infant was visited at home late afternoon or early evening. It was weighed naked, and a soft probe inserted $5 \mathrm{~cm}$ from the anal margin to monitor rectal temperature by a Grant Squirrel Data logger set to sample once a minute throughout the night. We have used rectal probes to monitor the development of body temperature patterns in over 500 babies over a period of 14 years with no adverse effects. Parents very rarely raise any objections. A second probe connected to the same logger monitored room temperature. Each infant was also connected to a Nellcor NL200 pulse oximeter through a toe probe. Electrocardiograph electrodes were attached, so that the oximeter output would reliably indicate heart rate. Heart rate was monitored from bedtime until the first morning feed. Monitoring continued during night time feeds or changes, and parents were instructed on the refitting of the oximeter probe. Each night parents were given a diary to record, prospectively, all events affecting the baby, such as feeds, nappy changes, periods of disturbance, etc. A second portion of the diary allowed the recording of the baby's condition over the days preceding recording, so that periods of illness 


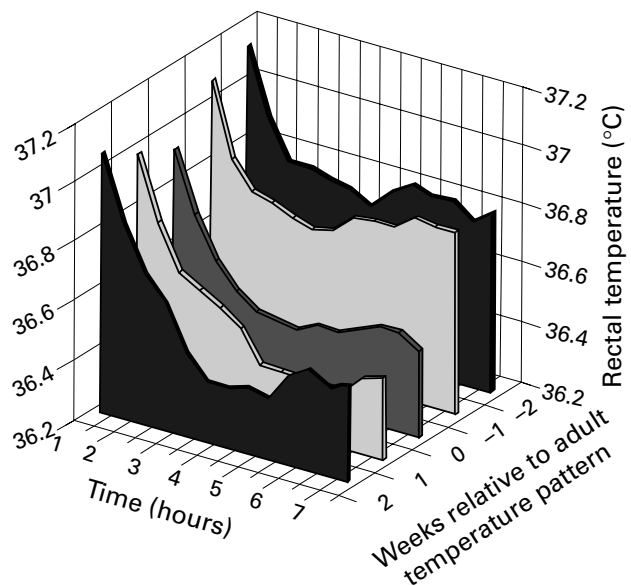

Figure 1 Development of night time temperature patterns. Mean rectal temperature is shown of 119 infants at half hourly intervals for eight hours from bedtime for two weeks before and three weeks after the week in which the sleeping rectal temperature first fell below $36.5^{\circ} \mathrm{C}$ for at least one hour during the night. Standard errors are not shown for reasons of clarity, but are typically $0.03-0.05^{\circ} \mathrm{C}$. There were no significant differences from week to week before and week to week after the adult-like pattern appeared, but a highly significant (Student's $t$ test $t=7.96 ; 118 \mathrm{~d}$; $p<0.001)$ change at the week of its appearance.

could be recorded. Many babies were also monitored on the night after immunisation against diphtheria, pertussis, and tetanus.

After the monitoring period, the loggers were collected and data downloaded to a computer for analysis. Temperature data were scrutinised carefully for problems such as lost probes, and only unblemished data analysed further. Heart rate data were also carefully scrutinised, and then for the purposes of this paper the rate readings were averaged over five minutes around each half hour during the night, starting with time 0 at bedtime. More detailed analysis of minute to minute heart rate variation will be presented later. Curves of temperature and heart rate were compared by Student's $t$ test, paired when appropriate.

\section{Results}

SUBJECTS

Just over half $(63 ; 53 \%)$ of the 119 subjects were boys. Mean (SD) birth weight was 3276 (545) g, mean (SD) gestation was 39.1 (2.17) weeks, and mean (SD) maternal age was 26.4 (4.88) years. Some $71(60 \%)$ of the babies were bottle fed at 6 weeks. The social class distribution reflected that of the Leicestershire population with $20(17 \%)$ in social classes I and II and $44(35 \%)$ in social classes IV and V.

A total of 640 successful overnight recordings of heart rate were analysed, which averages 5.4 per infant. Six or more successful consecutive weekly recordings were made in 49 (41\%) infants around the age at which an adult-like body temperature pattern appeared.

BODY TEMPERATURE

The cohort of infants taking part in this study developed night time body temperature patterns as we have described previously. For the early weeks of monitoring, body temperature fell to approximately $36.8^{\circ} \mathrm{C}$ with sleep at night. This pattern did not change from week

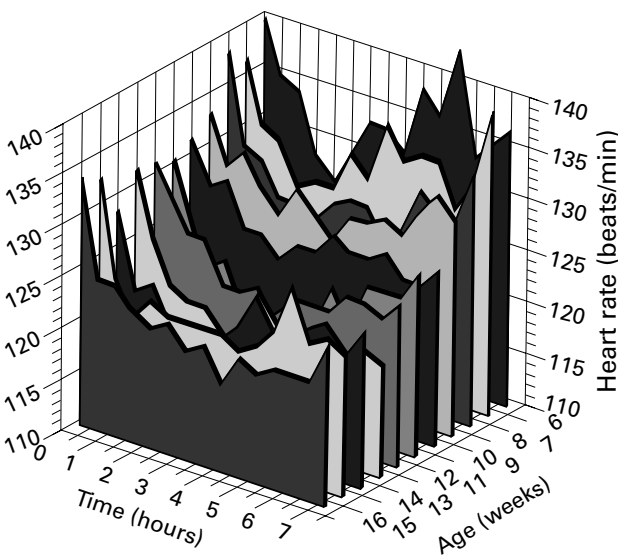

Figure 2 Overnight patterns of heart rate at different ages. Heart rate is plotted at half hourly intervals from bedtime in 119 babies of 7-16 weeks of age. Standard errors are not shown for reasons of clarity, but are typically 2-3 beats/min. There was a significant fall in heart rate with age, until about 14 weeks.

to week for a variable number of weeks until, suddenly, between one week and the next the pattern changed. Body temperature fell to approximately $36.3^{\circ} \mathrm{C}$. Once established, this pattern was maintained, essentially unchanged, from week to week unless the baby became ill or was immunised. Figure 1 illustrates this process. Data from all the infants have been normalised to the first week in which the minimum night time sleeping body temperature fell below $36.5^{\circ} \mathrm{C}$ which is week 0 . This occurred at ages ranging from 7 to 17 weeks in different babies. There were no significant differences from week to week before this week 0 , but a highly significant difference (Student's $t$ test, $t=7.96 ; 118 \mathrm{df} ; \mathrm{p}<0.001$ ) between that week and the previous week. Thereafter there were no significant differences from week to week. It was always possible to identify the week in which the adult-like body temperature pattern first appeared.

CHANGES IN PATTERN OF HEART RATE WITH AGE Figure 2 shows a cross sectional analysis of the heart rate data by age. Both the mean heart rate over the night (from bedtime until eight hours later) and the minimum sleeping heart rate apparently fell steadily until about 14 weeks of age, and remained stable from week to week thereafter. There was a highly significant change with age $(t=6.01 ; 118 \mathrm{df} ; \mathrm{p}<0.001$ comparing 8 weeks with 14 weeks). This gradual change was, however, an artefact of data collation. Figure 3 shows individual patterns of minimum sleeping heart rate with age, plotted alongside changes in the minimum sleeping body temperature. In these cases, both girls (see below) there was a sudden change in heart rate of between 5 and 8 beats/min at the time that the minimum body temperature suddenly fell. The age of this transition correlated closely with the age at which the adult-like body temperature pattern appeared, and therefore varied from 7 to 17 weeks.

Figure 4 shows heart rate data normalised in the same way as the temperature data from the same infants in fig 1 . In the population as a whole, the sleeping heart rate fell significantly 

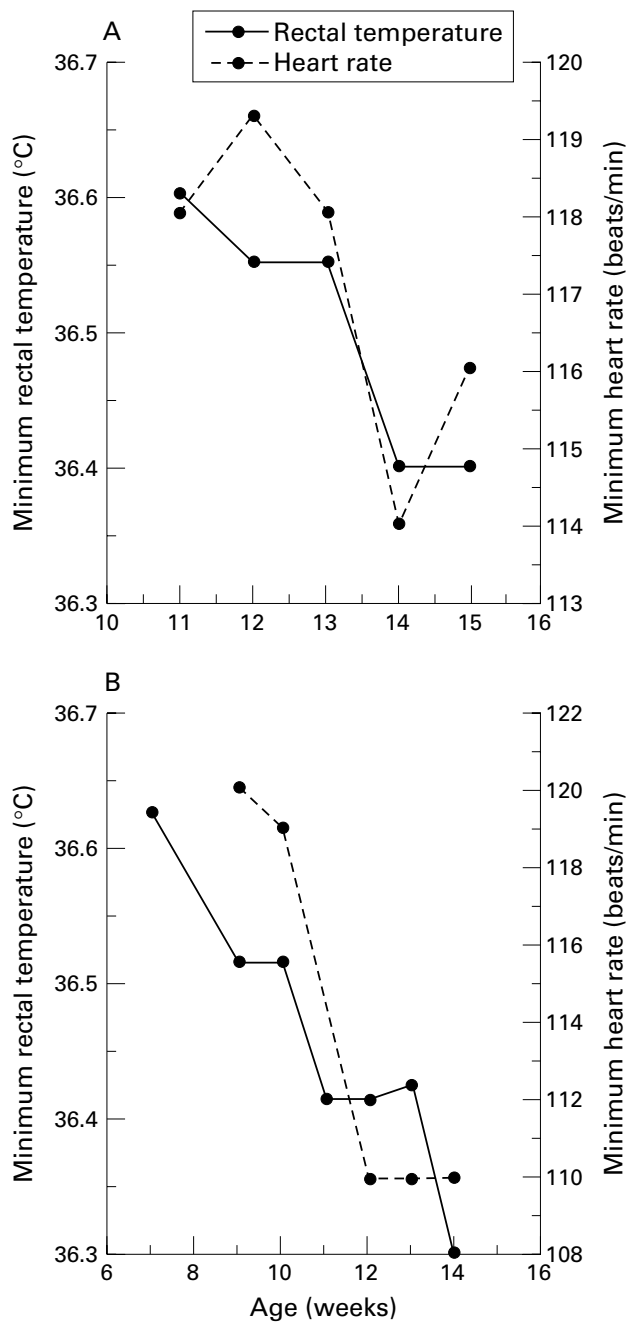

Figure 3 Minimum sleeping body temperature and minimum sleeping heart rate plotted as a function of age in two girls who attained the adult-like body temperature pattern at different ages.

$(t=4.98 ; 118 \mathrm{df} ; \mathrm{p}<0.001)$ between two weeks before and one week after the adult-like pattern was established, and then there was a further significant fall $(t=4.40 ; 118 \mathrm{df}$; $\mathrm{p}<0.001$ ) between one week before and the week that the adult-like pattern appeared.

SEX DIFFERENCES IN THE RELATION BETWEEN HEART RATE AND BODY TEMPERATURE PATTERNS The apparent two stage change in heart rate pattern around the time of onset of the temperature rhythm can be explained if data are collated separately for the two sexes. Figure 5 shows the minimum sleeping heart rate (between two and five hours after bedtime) in the weeks before and after the adult-like temperature pattern appeared, separately for boys and girls. In boys, the heart rate fell between two and one week before the adult-like temperature pattern developed but then did not change significantly from week to week. In girls, heart rate did not fall until the adult-like temperature pattern appeared, then reached a significantly lower value than the boys. Before the adult-like temperature pattern, boys had a higher heart rate than girls, although the difference barely reached statistical significance.

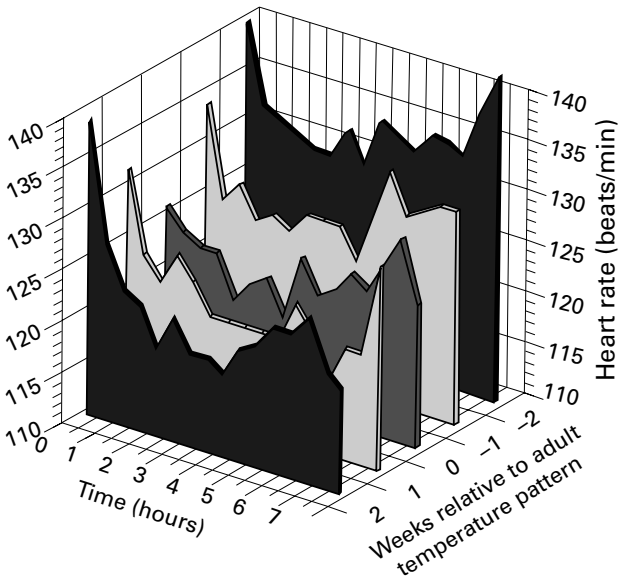

Figure 4 Overnight pattern of heart rate before and after the appearance of an adult-like night time body temperature pattern. The mean heart rate of 119 babies is plotted at half hourly intervals from bedtime for two weeks before and three weeks after an adult-like night time body temperature pattern first appeared. Heart rate fell significantly between two weeks and one week before $(t=4.98 ; 118 d f ; p<0.001)$, and then fell significantly again between the week before and the weeks of attainment of the adult-like pattern $(t=4.40 ; 118 d f ; p<0.001)$.

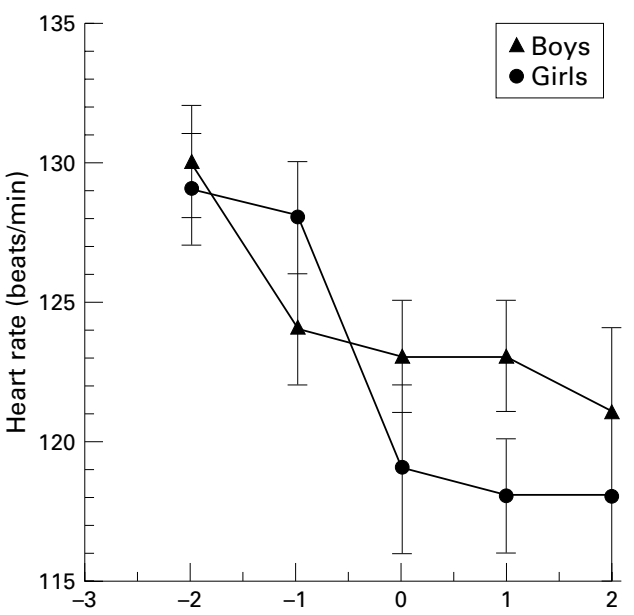

Age relative to adult-like temperature pattern

Figure 5 Sex differences in heart rate patterns. The mean heart rate between one and three hours after bedtime is plotted for two weeks before and three weeks after the adult-like night time temperature pattern first appeared. In both boys and girls there was a significant fall in heart rate $(t=2.42 ; 117 d f ; p<0.05)$ between two weeks before and one week after the adult-like temperature pattern. The heart rate of boys, however, changed more gradually, and at one week after the adult-like temperature pattern, the heart rate of boys was significantly higher than that of girls.

One week after the adult-like pattern had appeared, however, girls had a significantly $(t=2.42 ; 117 \mathrm{df} ; \mathrm{p}<0.05)$ lower heart rate than boys.

HEART RATE AND PARENTAL SMOKING

At each visit, parents were asked whether they smoked. Data were then collated separately for babies for whom one or more parents smoked and those for whom neither parent smoked. The analyses were also performed separately for infants before and after the adult-like body temperature pattern appeared. Before the adult-like pattern appeared, there was no significant effect of parental smoking on the night time heart rate pattern of infants, but 


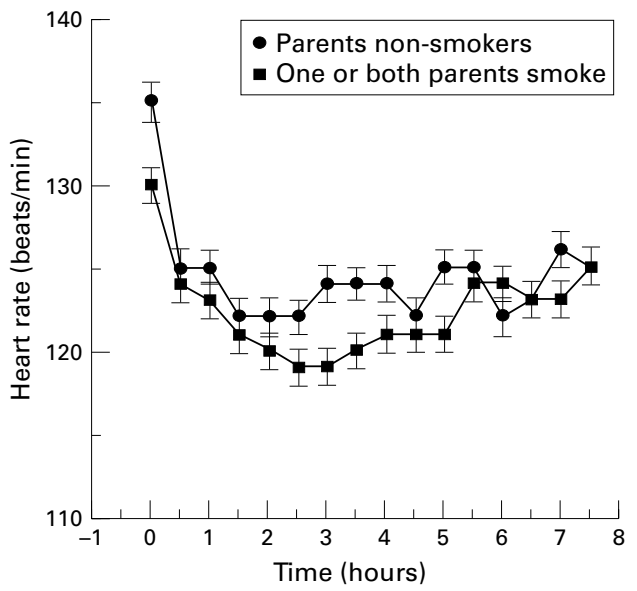

Figure 6 Effects of parental smoking on sleeping heart rate in infants who have developed an adult-like night time body temperature pattern. If one or both parents smoked, babies had a significantly $(t=5.76 ; 117 d f ; p<0.01)$ lower heart rate.

once the adult-like pattern had appeared the infants of smoking parents reached significantly lower heart rates (fig 6$)(t=5.76 ; 117$ df; $\mathrm{p}<0.01)$.

\section{Discussion}

We have shown that a detailed longitudinal study of individual infants is essential if the precise nature of developmental changes in sleeping heart rate pattern is to be shown. Although a cross sectional analysis such as that in previous studies indicates, as previously reported, an apparently gradual fall in sleeping heart rate from 6 to about 14 weeks of age, this is an artefact of averaging together individual developmental patterns which consist of a sudden change from one heart rate pattern to another at different ages in different babies. This sudden transition is associated with a similarly abrupt transition between two different patterns of sleeping body temperature.

The age of transition varies from infant to infant. It may be as young as 7 weeks or as old as 17 weeks in this cohort of infants. This means that some babies may maintain a relatively high heart rate (around 130 beats/ $\min$ ) for up to twice as long as others. The consequences of this are not obvious, but in other studies we have shown that babies with later development of an adult-like body temperature pattern share some characteristics with infants at increased risk of sudden death in infancy. ${ }^{23}$
The relation between developmental changes in heart rate and temperature is different in boys and girls. The heart rate of boys falls before an adult-like body temperature develops, and to a lesser extent than that of girls, who show an abrupt, relatively large fall (around 10 beats/min) when they develop an adult-like body temperature pattern. We can, at present, offer no explanation for these differences, except that boys do tend to develop adult-like temperature patterns about one week later than girls, which means that, on average, but not of course individually, boys and girls achieve the more mature heart rate pattern at the same age. Boys tend to gain weight faster, which may be a reason why their metabolic rate and perhaps therefore their heart rate cannot fall as much as girls.

The effect of parental smoking on sleeping heart rate was both unexpected and inexplicable. Why heart rate should be lower in passive smoking infants we cannot say. Perhaps the function of the autonomic nervous system in these infants is altered by nicotine.

Surprisingly, we found no evidence for a difference in heart rate between bottle fed and breast fed babies at any stage of development. Had there been such a difference, it might have offered some explanation for the sex differences (boys being more likely to be breast fed than girls) and the apparent effects of smoking (parental smoking is well correlated with bottle feeding).

We have, however, shown substantial individual differences in the development of patterns of sleeping heart rate that warrant further investigation.

We thank the Foundation for the Study of Infant Deaths for support.

1 Lodemore MR, Petersen SA, Wailoo MP. The development of night time temperature rhythms over the first six months of life. Arch Dis Child 1991;66:521-524.

2 Lodemore MR, Petersen SA, Wailoo MP. Factors affecting the development of night time temperature rhythms. Arch Dis Child 1992;67:1259-61.

3 Petersen SA, Wailoo MP, Williams SR. Low apgar scores are correlated with delayed development of circadian rhythms of body temperature in babies. F Physiol 1993;467:294.

4 Harper RM, Toke Hoppenbrouwers MB, et al. Polygraphic studies of normal infanmts during the first six months of life. 1. Heart rate and variability as a function of state. Pediatr Res 1976;10:945-51.

5 Richards JM, Alexander JR, Shinebourne EA, et al. Sequential 22-hour profiles of breathing patterns and heart rate in 110 full-time during their first 6 months of life. Pediatrics 1984;74:763-7. 